Special issue of the 2nd International Conference on Computational and Experimental Science and Engineering (ICCESEN 2015)

\title{
Design and Prototype Manufacturing of a Torque Measurement System
}

\author{
D. KARAYEL ${ }^{a, *}$ AND V. YEGIN ${ }^{b}$ \\ ${ }^{a}$ Sakarya University, Technology Faculty, Mechatronic Engineering Department, Sakarya, Turkey \\ ${ }^{b}$ Mustafa Kemal University, Engineering Faculty, Mechanical Engineering Department, Hatay, Turkey \\ In the present day, automobile manufacturers demand a certain torque range from hinges provided by supply \\ industry. However, there are no torque measurement system used by the industry to measure this kind of mecha- \\ nisms and the available systems cannot meet expectations. The purpose of this study is to design a computer-based \\ torque measurement system and to produce its prototype. This system will be used to measure torque range of \\ the vehicle front hood hinges and to control whether torque value is suitable or not. The developed system is an \\ integrated mechatronic system that contains such subsystems as mechanical, electronic and control system. It is \\ expected that the developed torque measurement system will fill an important gap in the relevant industrial sectors \\ and will find widespread application area.
}

DOI: 10.12693/APhysPolA.130.272

PACS/topics: 07.10.Pz, 83.85.Ns

\section{Introduction}

The increasing high performance and comfort expectations make the automotive manufacturers and the subindustry to seek new systems and manufacturing techniques. Within the scope of the mentioned developments, today, the opening and closing torque value of the hinged moving parts in vehicles is asked to be in a certain range. Also, supply industries have to present torque test reports together with the delivered hinges. However, at present, there is not any torque measurement system, used by the sub-industry, capable of fulfilling the expectations. This necessity is an important problem in supply industries. In fact, similar problems related to the torque measurement have been experienced in other industries. Therefore, researchers have studied different torque measurement systems in various industries, and they have developed specific torque measurement system for each sector [1-4]. In some studies, wireless measurement systems for measurement of mechanical torque have been developed $[5,6]$.

In this study, the computer-based torque measuring system has been developed for automobile hinges. Torque values of automobile hinge can be monitored and controlled in real-time by using the developed system. The integration of the system has been ensured by a designed mechanical part and a control system, and then manufacturing of the prototype has been carried out. Finally, the study has been completed by testing and verification processes.

\section{Design and manufacture of the system}

Nowadays, certain ranges of the opening and closing torque value of the hinges used in the automotive industry is one of the important features desired by the

\footnotetext{
* corresponding author; e-mail: dkarayel@sakarya.edu.tr
}

industry's leader companies. However, a special torque measurement system developed to measure the torque of the automotive hinge, which would be available on the market, could not be found within the scope of our literature research. The torque measurement system discussed in this study is a subsystem of an extensive project called "Hinge mounting counter mechatronic system design and prototype manufacturing". Therefore, the primary requirement of the measuring system is that it would be compatible and could work synchronously with the main system. In this case, it is necessary to make a special design of the measurement system. Mounting table with such system and an example of a hinge, the torque of which can be measured on this system, are shown in Fig. 1.

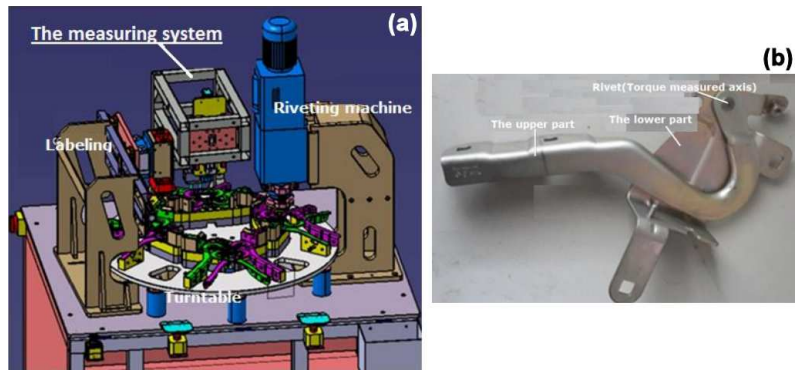

Fig. 1. (a) Hinge mounting table and (b) example of the measured hinge.

The measuring system is an integrated mechatronic system that contains the mechanical, electrical and control systems. The main parts of the system include a $\mathrm{PC}$, precision servo motor, high accuracy torque sensor, motion control couplings, ball bearings, pneumatic piston, DC power supply, DAQ card and RS485 transceiver. The implementation of the study in the form of block diagram is shown in Fig. 2. 


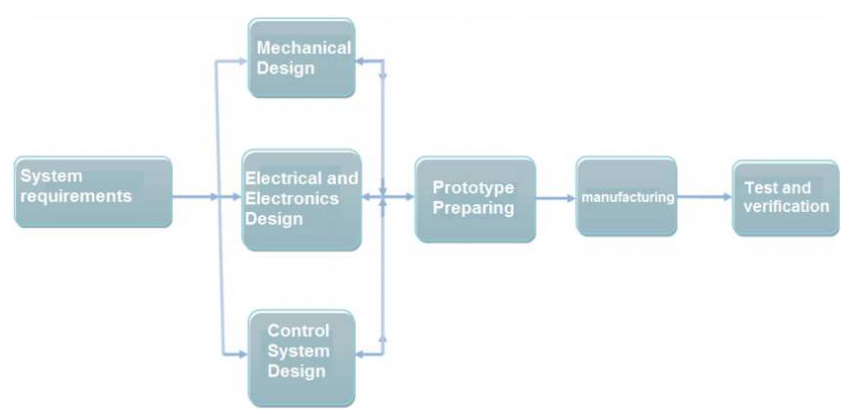

Fig. 2. System architecture. Mechatronic system approach.

The design of the torque measuring system has begun with the determination of system requirements. Then, the design of the mechanical system, electrical and electronic system and the control system have followed. Each of the three different designs has been carried out simultaneously at this stage. Finally, the study was completed with preparation of a prototype, manufacturing, testing and verification processes, respectively. The general functional view of the prototype of the measurement system, composed of many subsystems, is shown in Fig. 3.

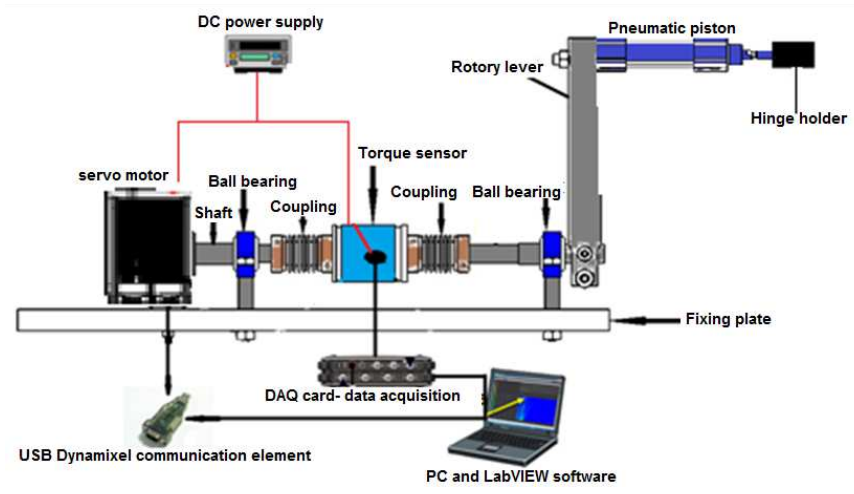

Fig. 3. The general view of the torque measuring system.

\subsection{Mechanical design}

The instrument designer's task is to find the best compromise between cost and benefit to the users. The mechanical design of the system has been carried out in two stages of conceptual design and the detailed design. In the conceptual design stage, the main specifications of the system were determined. The conceptual design stage is an attempt to find the optimal system. At this stage, the measuring system was analysed and evaluated according to function, quality level, cost and even ergonomic-aesthetic conditions. After the conceptual design phase, all studies until the manufacturing of the system have been carried out in the detailed design stage. The detailed design stage contains mainly such activities as preparing the kinematic scheme, determining of the standard and available components, systems analysis and dimensioning, preparation of drawings. The critical elements of the system have been also analysed in terms of strength, structural deformation and dynamic behaviour in the detailed design stage. Following these mechanical design studies, the mechanical design and prototype of the system have been developed as shown in Fig. 4.
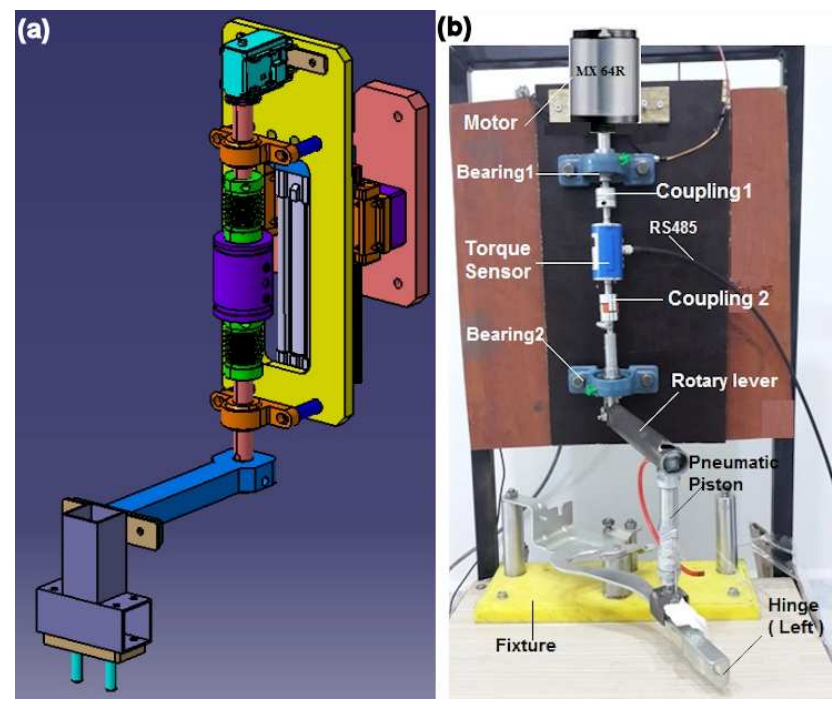

Fig. 4. The mechanical design and the prototype of the measuring system. The final mechanical design. The prototype photo.

The dynamic modelling of the system is important from a scientific perspective. Therefore, here, the modelling of the mechanical structure of torque measuring system will be discussed in more detail. For modelling of the system, the mechanical structure of the measuring system has been represented as a chain system, made of undeformable bodies and massless elastic elements. Here, the generalized coordinates correspond to angular positions of the respective non-deformable elements. The developed model is presented in Fig. 5.

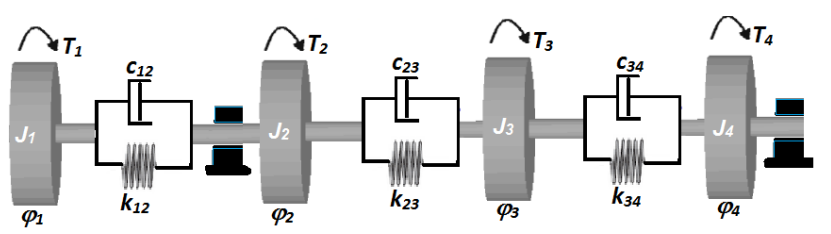

Fig. 5. The dynamic model of the measuring system.

In Fig. 5, $\phi_{i}$ is the angular position of the nondeformable elements (generalised coordinate), $T_{i}$ torque, $J_{i}$ moment of inertia of the non-deformable elements, $c_{i}$ coefficient of viscous damping, $k_{i}$ torsional stiffness of the deformable elements.

The model can be expressed by the following differential equation in the matrix form:

$$
[J]\{\ddot{\varphi}\}+[C]\{\dot{\varphi}\}+[K]\{\varphi\}=[T]
$$

Here, the free vibration equation can be written as follows: 


$$
[J]\{\ddot{\varphi}\}+[C]\{\dot{\varphi}\}+[K]\{\varphi\}=0,
$$

where, $[J]$ is the diagonal inertia matrix, $[K]$ stiffness matrix, $[C]$ damping matrix, $\{\varphi\}$ angular displacement vector, $\{\dot{\varphi}\}$ angular velocity vector and $\{\ddot{\varphi}\}$ is angular acceleration.

In systems with steel couplings the damping is small and has little effect on the natural frequencies. Therefore, the torsional natural frequencies and mode shapes may be calculated assuming that the damping is zero. The equation can be simplified, since the damping matrix $[\mathrm{C}]=0$. Also, the dynamic behaviour of the system has been assumed to be a simple harmonic motion $(\varphi=\varphi \sin \omega t)$. According to this approach, the solution of the matrix equation can be written as follows

$$
[J]^{-1}[K]\{\varphi\}-\left[\omega^{2}\right]\{\varphi\}=0
$$

This form of the matrix equation is known as the eigenvalue equation. The values of $\omega^{2}$, for which the equation is solvable, are known as the characteristic values or eigenvalues of the matrix. Correspondingly, the vector solutions for $\{\varphi\}$ are the eigenvectors of the matrix $[J]^{-1}[K]$, which are referred to as the stiffness-mass matrix. This equation can be solved when the determinant of the coefficient matrix is equalized to zero. The equation obtained from the determinant of the coefficient matrix is known as the characteristic equation of the matrix. The values of $\omega$ in the equation are the eigenvalues. In this context, the stiffness and/or mass of the system elements have been adjusted to appropriate values and thus it has been assured that the resonant frequencies of the developed system remain out of the critical values.

\subsection{The control system}

The main tasks of the control system are to control the servo motor, to monitor the process and to record the measurement results. The system mainly consists of servo motor, DAQ card, torque sensor and a computer with LabVIEW. The control system block diagram is shown in Fig. 6 .

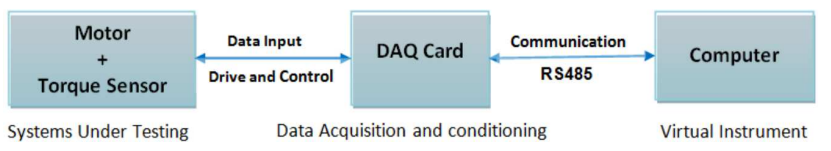

Fig. 6. The block diagram of torque measuring system.

LabVIEW software has been preferred for performing all control functions as a real-time computer-based system. DAQ card has been used for data transfer. The PC program flow chart is shown in Fig. 7. As shown in the flow chart, the control process begins with the initialization of devices via the LabVIEW interface. The next process is to load input parameters. In the following step, the hinge is moved by the servo motor according to the input parameters and the torque sensor measures torque value of the hinge during movement. Then, the data acquisition and processing are realized by the control software.
Finally, the control process is completed by displaying, storing and transferring the data.

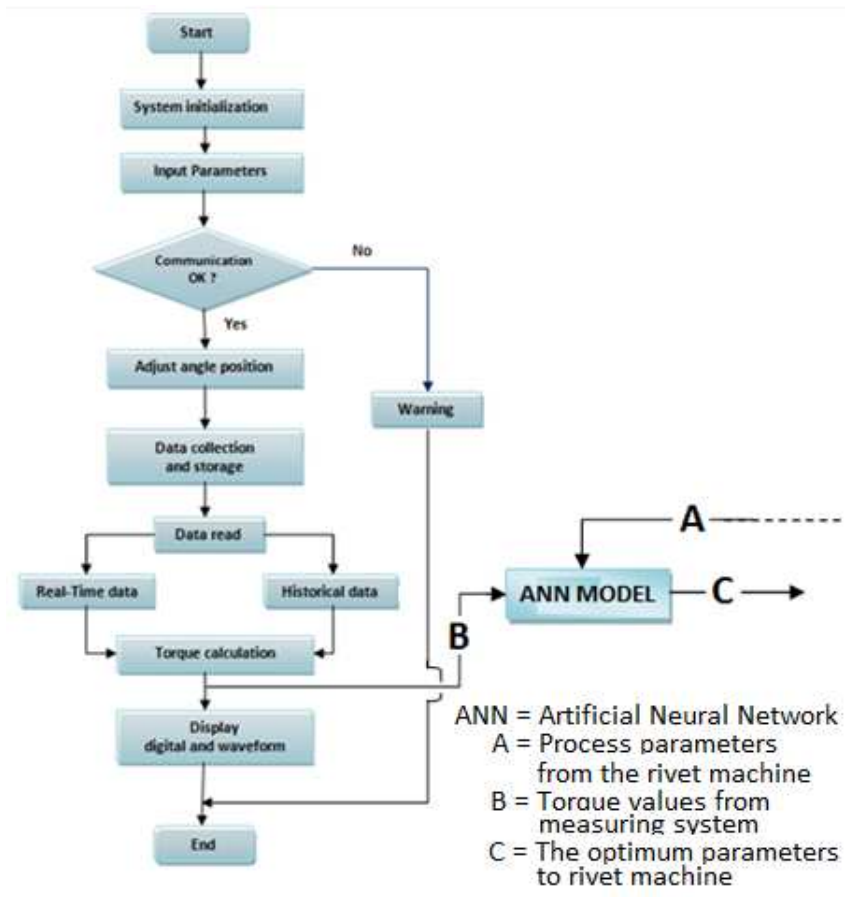

Fig. 7. PC program flow chart.

\section{Manufacturing, mounting and verification}

Primarily, the shafts have been connected with the servo motor, the rolling bearings, the torque sensor and the couplings. In the assembly, maximum attention has been dedicated to providing the geometric tolerances. A lot of hinges, for which there are known torque values, have been measured again in order to verify the developed system. Graphical user interface with digital and graphic display of measurement results is shown in Fig. 8. Eventually, the system has successfully passed a series of performance tests and has been registered as a professional tool in the industry.

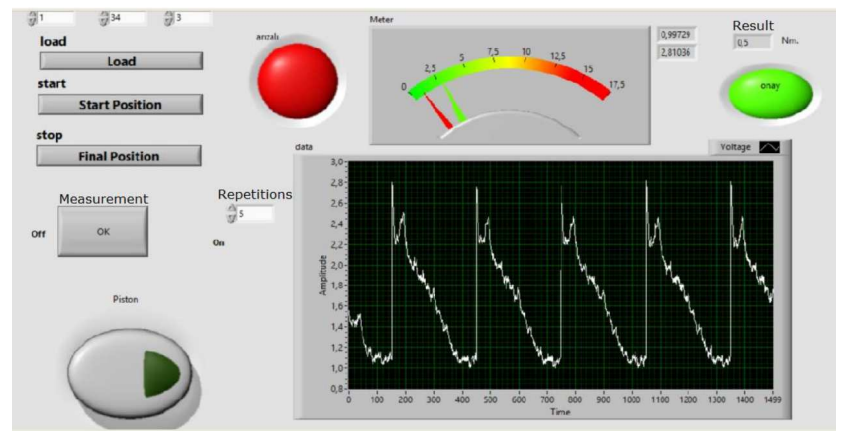

Fig. 8. LabVIEW graphical user interface together with the measurement results. 


\section{Conclusions}

The following main conclusions can be generally drawn from the study:

- The torque measurement of hinges of passenger cars has begun recently to be demanded by the automotive industry. However, the available torque measurement systems do not fully meet the needs in this area. The developed system is one of the few systems designed specifically for this field. It is relatively new in this area. For now, it is designed only for a specific brand (Renault front hood hinge). However, the system can be used for different brands/models in the future, with minor modifications. In this regard, the system is relatively novel and original.

- As mentioned previously, the torque measuring system is a subunit of the integrated rotary table system used to mount hinges. The rotary table consists of such subsystems as assembly unit, measurement unit and labelling unit and it works precisely at high speed. The developed torque measurement system has to work simultaneously and to be compatible with the integrated system. Therefore, it is very important to select the components and operating conditions of the measuring system so that it can provide the expected performance. Thus, the system has been designed using advanced technology equipment and by taking into account the results of dynamic analysis. This can be considered as an advantage of this system in comparison with ordinary similar systems.

- The system has a friendly interface. It can be said that easy and stable operation, high accuracy, low cost, flexibility, short development cycle, light weight, easy installation are the most significant advantages of the developed system.

- Another advantage of the developed system is that the measurement results can be stored, organized in a report and can be optionally transferred to other units of the integrated system.

- The developed system can be also used to determine the angular stiffness of the available mechanical structures. In this aspect, this study will also make a significant contribution to other researchers, whose work is related to structural analysis.
- It is also expected that the study presented in this paper will provide a basis for the design and development of subsequent intelligent measuring systems. In this context, LabVIEW and artificial neural networks will be integrated and used together for the optimization process in future.

- In this study, it is aimed to determine optimum process parameters corresponding to the desired torque values of the hinge for the riveting. This available study will be a basis for the future optimization study. The developed LabVIEW-based platform and artificial neural networks will be utilized together in the future study. We also hope that this integration will be original and interesting.

It is expected that the developed torque measuring system will have a widespread usage area by filling an important gap in the industry.

\section{Acknowledgments}

This study was supported by the master thesis project no. 2014-50-01-037 and Scientific Research Project no. 2015-09-18-001 by Sakarya University Department of Scientific Research Commission and Republic of Turkey Ministry of Science, Industry and Technology and by Industrial Thesis Supporting Program (SAN-TEZ) with project coded 0409.STZ.2013-2. We would like to express our thanks to Sakarya University and Ministry of Science, Industry and Technology for their support.

\section{References}

[1] M. Köster, M. Thommes, Chem. Eng. J. 164, 371 (2010).

[2] M. Chandra Sekhar Reddy, A.S. Sekhar, Measurement 61, 111 (2015).

[3] W.F. Traoré, R. McCann, in: Power and Energy Society General Meeting (PES), IEEE, Vancouver 2013, p. 1.

[4] Danfeng Chen, Aiguo Song, Ang Li, Procedia Eng. 99, 1164 (2015).

[5] V. Niskanen, J. Ahola, in: Proc. Power Electronics and Applications (EPE 2011), IEEE, Birmingham 2011, p. 2974.

[6] L. Baghli, J.F. Pautex, S. Mezani, in: Proc. XIX International Conference on Electrical Machines(ICEM 2010), IEEE, Rome 2010, p. 1. 